\section{Síndrome de Bayés en el perioperatorio}

\author{
Bayes syndrome in the perioperative period
}

\author{
Acad. Dr. Raúl Carrillo-Esper,* Dra. Dulce María Carrillo-Córdova ${ }^{\ddagger}$
}

Citar como: Carrillo-Esper R, Carrillo-Córdova DM. Síndrome de Bayés en el perioperatorio. Rev Mex Anestesiol. 2021; 44 (4): 311-313. https://dx.doi.org/10.35366/100878

\begin{abstract}
RESUMEN. Los bloqueos auriculares se caracterizan por alteraciones en la conducción secundarias a un retardo 0 bloqueo en el haz de Bachmann. El Dr. Antonio Bayés de Luna fue uno de los primeros en describir de manera extensa esta entidad a partir de 1979, clasificándolo en inter- e intraauriculares. El bloqueo interauricular se caracteriza en el electrocardiograma (ECG) por onda P con duración mayor a 120 mseg y que presentan morfología bimodal, especialmente en las derivaciones DI, DII, aVL y en las derivaciones inferiores. Existen varios tipos y grados de bloqueo interauricular relacionados a la magnitud del deterioro de la conducción entre las aurículas. Este bloqueo se asocia con frecuencia a taquiarritmias, en especial fibrilación auricular. El manejo incluye antiarrítmicos, anticoagulantes y, en casos especiales, terapia de resincronización auricular. El objetivo de este trabajo es enfatizar en la importancia de la evaluación de la onda P y de los bloqueos interauriculares en el período perioperatorio.
\end{abstract}

ABSTRACT. The interatrial block is an auricular conduction abnormality secondary to delay or block through the Bachmann's bundle. Dr. Antonio Bayés de Luna was the first who provided a clear description of atrial conduction block in 1979, classifying them into either inter- and -intra atrial. The interatrial block is expressed in the electrocardiogram (ECG) by the presence of $P$-wave duration that equals or exceeds 120 mseg and presents usually a bimodal morphology, especially in leads I, II, aVL and inferior leads. There are different types of interatrial block related to deterioration of conduction between the right and left atrium. It was demonstrated that this type of block is very frequently accompanied by paroxysmal atrial arrhythmia, especially atrial fibrillation. Current medical therapies included anti-arrythmic, anticoagulation and in special cases atrial resynchronization. The aim of this paper is to emphasize the importance of the evaluation of $P$ wave and interatrial blocks in the perioperative period.

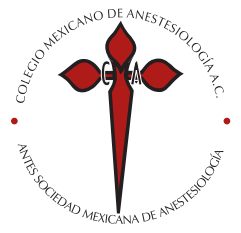

Palabras clave: Bloqueo interauricular, síndrome de Bayés, onda P.

Keywords: Interatrial block, Bayes syndrome, $P$ wave.

* División de Áreas Críticas. Instituto Nacional de Rehabilitación «Luis Guillermo Ibarra Ibarra».

${ }^{\ddagger}$ Residente de Medicina Interna. Instituto Nacional de Ciencias Médicas y Nutrición «Salvador Zubirán».

Correspondencia: Dr. Raúl Carrillo-Esper Instituto Nacional de Rehabilitación Luis Guillermo Ibarra Ibarra Calz. México-Xochimilco No. 289, Coapa, Arenal Tepepan, Tlalpan, 14389, CDMX

E-mail: raulcarrilloesper@gmail.com

Recibido: 14-02-2020

Aceptado:28-02-2020

\section{INTRODUCCIÓN}

L a fibrilación auricular (FA) es una de las arritmias más fre$\checkmark$ cuentes en el adulto mayor y tiene una elevada prevalencia; proyecciones actuariales alertan sobre su incremento debido al envejecimiento de la población y su relación con enfermedades como la diabetes mellitus, la hipertensión arterial, la cardiopatía isquémica, el remodelamiento auricular, entre otras. La importancia de la FA reside en que es un factor de riesgo de cardioembolismo, además de las alteraciones hemodinámicas que condiciona, en particular cuando es muy rápida.

La evaluación del electrocardiograma en el preoperatorio es prioritaria, en especial en adultos mayores, pacientes con antecedentes de diabetes mellitus, hipertensión arterial o algún tipo de cardiopatía. Su revisión deberá ser integral para la detección de alteraciones del ritmo, la conducción, isquemia miocárdica, crecimiento de cavidades, etcétera; derivado de esto se puede estratificar el riesgo, además de dar continuidad de la atención en relación a una evaluación más completa y la implementación de una estrategia terapéutica.
Las alteraciones en la onda P son poco evaluadas y traducen una anormalidad electrofisiológica y estructural considerables, de las cuales destacan el bloqueo intraauricular, interauricular y la remodelación auricular. La trascendencia de los bloqueos interauriculares (BIA) radica en su estrecha relación con el desarrollo de FA.

El objetivo de este reporte es resaltar la importancia de la evaluación de los BIA en la evaluación preoperatoria, en especial en pacientes de alto riesgo y revisar conceptos actuales relacionados a esta importante alteración electrofisiológica.

\section{PRESENTACIÓN DE CASO}

Paciente de 68 años con antecedentes de hipertensión arterial sistémica tratada con amlodipino y diabetes mellitus de 10 años de evolución manejada con dieta e hipoglucemiantes orales. Refiere que cursa con cuadros intermitentes de palpitaciones que no han sido evaluadas. Ingresó al hospital por fractura de cadera derecha secundaria a caída de su propia altura. En el electrocardiograma se observó ritmo sinusal, con 
onda P con duración entre 110 a 120 mseg, y ondas P bifásicas y bimodales (Figura 1). En el ecocardiograma se observó aurículas normales, TAPSE 21, septum interauricular íntegro, no trombos ni masas intracavitarias. Válvulas sin alteraciones. Ventrículo derecho normal. Ventrículo izquierdo con remodelación concéntrica, masa miocárdica de $90 \mathrm{~g} / \mathrm{m}^{2}$. Fracción de expulsión con técnica de Simpson de 67\%. Función diastólica con patrón de relajación lenta. Pericardio sin alteraciones. En el caso que nos ocupa se observaron cambios en las ondas $\mathrm{P}$ caracterizadas por duración de 120 mseg, ondas P bimodales en derivaciones inferiores y en precordiales de V1 a V3 y ondas P bifásicas en DIII. Con el diagnóstico de síndrome de Bayés (SB) se inició tratamiento antiarrítmico con amiodarona y posterior a la cirugía con anticoagulación. No se presentaron taquiarritmias ni FA durante su estancia hospitalaria. Se dio de alta para continuar seguimiento por cardiología.

\section{DISCUSIÓN}

El conocimiento de los bloqueos auriculares no es reciente. Bachmann $^{(1)}$ fue el primero en describir los bloqueos interauriculares en 1916, para 1956 Bradley y Marriot ${ }^{(2)}$ reportaron en una serie de 4,500 electrocardiogramas una incidencia de bloqueos interauriculares de $4.5 \%$, definiéndolo como una $\mathrm{P}$ con duración de entre 110 a 120 mseg. Fauchier ${ }^{(3)}$ describió que esta entidad se puede asociar a otras alteraciones del ritmo y la conducción destacando la disfunción del nodo sinusal, alteraciones de conducción auriculoventricular, taquicardia paroxística supraventricular y fibrilación auricular paroxística, con incidencias que van de 16 a 31\%.

La prevalencia del BIA o SB es de edad dependiente, en grupos poblacionales $<20$ años es de $5.4 \%$, por arriba de los 60 años y con base en factores de riesgo puede llegar a ser de hasta $60 \%$ (como en el caso de foramen oval permeable e insuficiencia cardíaca), lo cual pudiese estar relacionado a inflamación y fibrosis auricular. De los factores de riesgo descritos destacan: hipertensión arterial, diabetes mellitus, hipercolesterolemia, cardiopatía chagásica avanzada, período postcardioversión farmacológica, postablación de fibrilación auricular y revascularización coronaria, entre otros ${ }^{(4-6)}$.

Para comprender el SB es importante recordar la actividad eléctrica auricular y la inscripción de la onda P. Fisiológicamente el impulso eléctrico se inicia en el nodo sinoauricular, de ahí viaja al nodo auriculoventricular a través de tres vías internodales que son: 1. Anterior, 2. Medio o de Wenckebach, 3. Posterior o de Thorel. Por el fascículo anterior o de Bachmann (puede viajar a través del seno coronario o la fosa oval) se lleva a cabo la conducción interauricular. Cuando el impulso eléctrico se bloquea en los tercios superior y medio del tabique interauricular en la región del haz de Bachmann se produce una activación retrógrada de la aurícula izquierda a través de conexiones adyacentes al seno coronario. El sustrato del SB se explica por los siguientes mecanismos: regulación a la baja de los canales de sodio, disfunción del mioplasma y de las uniones de los miocitos, actividad en exceso del estrés oxidativo, incremento en la resistencia axial y de la capacitancia de membrana, así como procesos amplificados por la inflamación y fibrosis. De esta manera el SB se manifiesta como una alteración en la inscripción de la onda P, caracterizada por su alargamiento entre 110 a 120 mseg, su morfología bimodal y/o bifásica que se manifiesta preferentemente en DI, DII, DIII y en V1 ${ }^{(7-10)}$.

\section{El bloqueo interauricular se clasifica en ${ }^{(11)}$ :}

1. Primer grado: se caracteriza por una activación normal de las aurículas, es decir, de derecha a izquierda, la P tiene
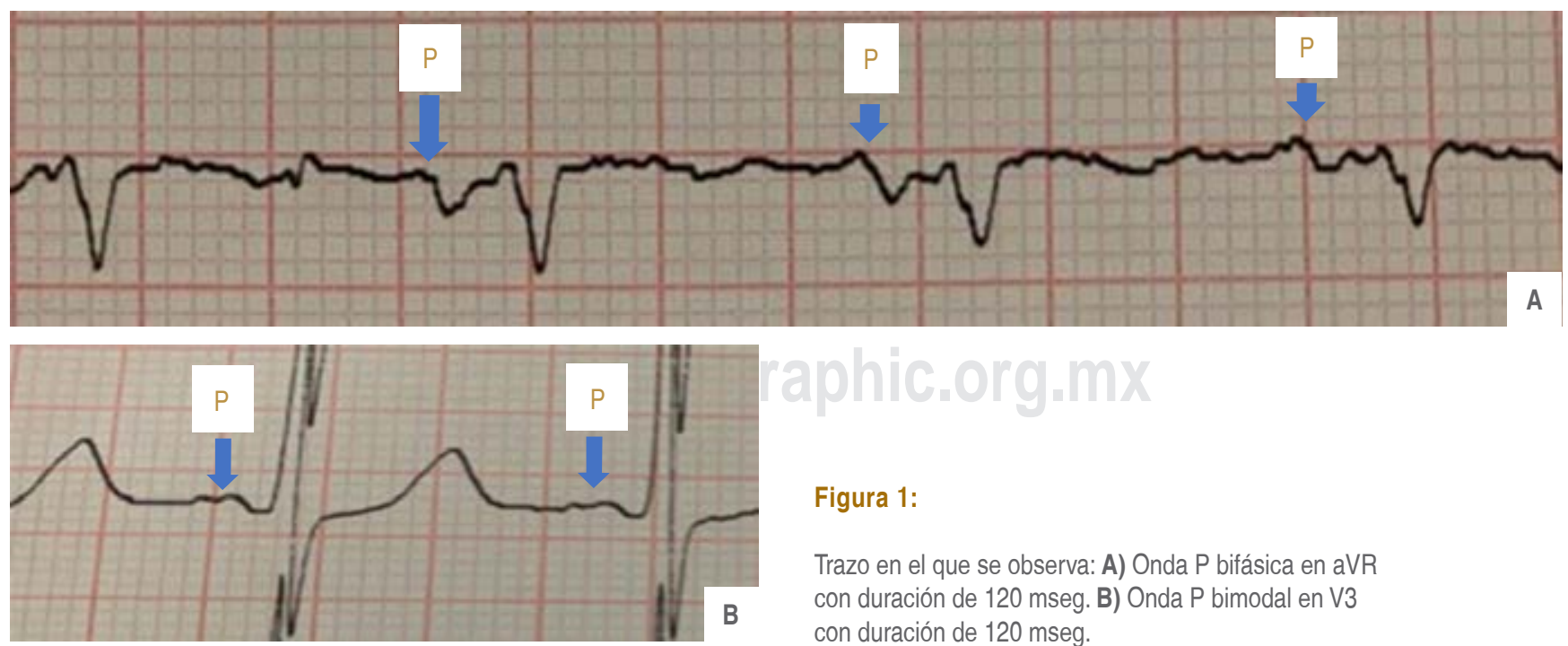

Figura 1:

Trazo en el que se observa: A) Onda P bifásica en aVR con duración de $120 \mathrm{mseg}$. B) Onda P bimodal en V3 con duración de 120 mseg. 
una duración de más de 120 mseg (algunos autores ponen en límite en $110 \mathrm{mseg}$ ) y con morfología bimodal en las derivaciones inferiores.

2. Segundo grado: se caracteriza por una onda P cambiante, que va de una morfología normal a patrón de primer grado, es decir, bimodal.

3. Tercer grado: se caracteriza por una activación anormal de las aurículas debido al bloqueo fascicular, ésta se lleva a cabo de manera retrógrada y a través del músculo adyacente al seno coronario. En el ECG se observa una P de más de $120 \mathrm{mseg}$, bimodal en DI y bifásica en las derivaciones inferiores (DII, DIII, aVF).

4. Variantes atípicas: se han descrito otras variantes del SB caracterizado por ondas $\mathrm{P}$ bifásicas en las derivaciones inferiores con duración de $120 \mathrm{mseg}$.

Es importante mencionar que el patrón electrocardiográfico puede ser cambiante, coexistir con otras alteraciones del ritmo (en especial con FA) y/o la conducción, así como coexistir con alteraciones auriculares, en especial crecimiento y remodelación de la aurícula izquierda. El SB se asocia clínicamente, y relacionado a la FA y desincronización auriculoventricular, a evento vascular cerebral de tipo cardioembólico, por lo que en estos pacientes se debe realizar un protocolo diagnóstico que incluya estudio Holter, ecocardiograma y, cuando amerite, resonancia magnética nuclear ${ }^{(12-15)}$.

En el caso que nos ocupa, el SB se fundamentó en la presencia de un bloqueo interauricular prolongado (P de $120 \mathrm{mseg}$ ), con onda P bimodal, en ritmo sinusal, esto junto con la presencia del componente final negativo de la onda $\mathrm{P}$ (onda bifásica) en una derivación inferior indicaban la activación de la aurícula izquierda en dirección caudocraneal secundario a un bloqueo avanzado del haz de Bachmann. Esta alteración electrofisiológica de seguro condicionaba FA paroxística, que se manifestaba con las palpitaciones intermitentes que refería el paciente. Es importante mencionar que en el ecocardiograma no se encontró crecimiento auricular o incremento en el volumen auricular que explicaran los cambios en la morfología de la onda P y el BIA.

\section{CONCLUSIÓN}

Al tomar en cuenta la elevada prevalencia del SB y su impacto clínico en los pacientes adultos mayores y de alto riesgo, el análisis del electrocardiograma (en especial de la onda P) debe ser parte integral del proceso de evaluación en el perioperatorio, en particular por ser marcador de arritmias supraventriculares, de las que destaca la fibrilación auricular, y por estar asociado a cardioembolismo.

\section{REFERENCIAS}

1. Bachmann G. The inter-auricular time interval. Am J Physiol. 1916;41:309-320.

2. Bradley SM, Marriot J. Intraatrial block. Circulation. 1956;14:10731078.

3. Fauchier JP, Charbonnier B, Latour F, Brochier M. Chronic idiopathic binodal block. Ocurrence, course and pathogenesis. Arch Mal Coeur Vaiss. 1979;72:1052-1058.

4. Jairath UC, Spodick DH. Exceptional prevalence of interatrial block in a general hospital population. Clin Cardiol. 2001;24:548-550.

5. Baranchuck A. Síndrome de Bayés: valor del electrocardiograma en la predicción de fibrilación auricular. Rev Urug Cardiol. 2015;30:404-410.

6. De Luna AB, Massó-van RA, Robledo LAE. The diagnosis and clinical implications of interatrial block. Eur Cardiol, 2015;10:54-59.

7. De Luna AB, Cladellas M, Oter R. Interatrial block and retrograde activation of the left atrium and paroxysmal supraventricular tachyarrtymias. Eur Heart J. 1988;9:112-115.

8. Conde D, Baranchuk A. Bloqueo interacuricular como sustrato anatómico-eléctrico de arritmias supraventriculares. Arch Cardiol Mex. 2014;84:32-40.
9. De Luna AB, Fort de Ribot R, Trilla E. Electrocardiographic and vectorcardiographic study of interatrial conductions disturbances with left atrial retrograde activation. J Electrocardiol. 1985;18:1-8.

10. Thomsen MB, Calloe K. Human atrial fibroblasts and their contribution to supraventricular arrhytmia. Physiol Rep. 2016;4:3-5.

11. Levinstein JM, Cal y Mayor MR, Ángel JVM. Bloqueo Interatrial (síndrome de Bayés). An Med (Mex). 2019;64:35-42.

12. Chhabra L. Importance of $P$ wave indices in stroke. Int J Cardiol. 2016;203:962-965.

13. Alexander B, Baranchuk A, Hasseb S, van Rooy H, Kuchtaruk A, Hopman W. Interatrial block predicts atrial fibrillation in patient with carotid and coronary artery disease. J Thorac Dis. 2018;10:4328-4334.

14. Benito EM, de Luna AB, Baranchuck A, Mont L. Extensive atrial fibrosis assessed by late gadolinium enhancement cardiovascular magnetic resonance associated with advanced interatrial block electrocardiogram pattern. Europace. 2017;19:377-380.

15. Enriquez A, Conde D, Villuendas R, Bayes-Genis A, de Luna AB, Baranchuk A. Interatrial Block and atrial fibrillation: invasive and noninvasive measurements may help to define the syndrome. Heart Rhythm. 2014;11:197-199. 